\title{
Assessing animal models of AIDS
}

\author{
Animal models of AIDS continue to surprise researchers with results that can be difficult \\ to explain. However, most researchers still believe that animal models are essential to \\ progress in understanding the disease. But which animal model is best? (pages 321-329)
}

A report published in this issue of Nature Medicine by Abimiku et al. asserts that vaccination with components of HIV-1 protects macaques against challenge with HIV-2 (ref. 1). This unexpected finding challenges many of our prejudices about how to vaccinate and protect against immunodeficiency viruses. When faced with surprising and possibly unwelcome news, there has been a tendency in AIDS research to 'shoot the messenger.' As a result, animal models have been condemned as unreliable, inappropriate and even misleading. The reality is that all animal models are potentially capable of yielding relevant information, provided that experiments are appropriately designed and well controlled. As in all scientific endeavour, individual observations need independent confirmation before they are accepted as established fact. If the results of in vivo experiments in different animal models appear to conflict with each other, with results of in vitro work or with accepted preconceptions, then this may reflect our ignorance of the biology of immunodeficiency viruses.

The observations reported by Abimiku et al. are intriguing, but essentially very preliminary. A group of three naive control animals is compared with eight vaccinated macaques, each of which received a unique immunization with one of several different cocktails of antigens. Hence, as the authors acknowledge, even when the disparate vaccinated individuals are treated as a single group, these results do not reach statistical significance and could be a consequence of chance. The report thus illustrates a common difficulty encountered with animal models for AIDS: the limited numbers of individuals allocated to test and control groups.

Four different types of model have been used in AIDS research: chimpanzees infected with HIV-1, macaque monkeys infected with either SIV or HIV-2, cats infected with feline immunodeficiency virus and SCID mice immunologically reconstituted with human cells and infected with HIV.

The use of non-human primates such as chimpanzees or macaques is constrained by generally accepted practical, economic

\section{James Stott \& NeIL Almond}

and even ethical limitations. As a result, undue scientific credibility has often been given to the protection against infection of very small numbers of non-human primates, numbers that defy the application of conventional statistical analysis. This has been particularly true of results obtained in chimpanzees. It is clearly important that results must be subjected to appropriate statistical analysis whenever possible and extreme caution exercised when this is not the case. However, more credibility is due where the studies form part of a logical sequence of investigation with a consistent theme and outcome. One solution to the problem has been to use a standardized stock of challenge virus

\section{When faced with surprising} and possibly unwelcome

\section{news, there has been a}

\section{tendency in AIDS research}

\section{to 'shoot the messenger.'}

\section{As a result, animal models}

\section{have been condemned .... .}

so that the accumulation of historical controls gives greater confidence in the validity of protected vaccinated individu$\mathrm{als}^{2}$. Most primate centres in the United States and Europe have been commendably generous in sharing well-characterized stocks of challenge viruses. Those working with macaques have also sought to pool resources and to design larger collaborative experiments using standardized vaccines and challenge viruses ${ }^{3}$. Nevertheless, the numbers of macaques that have been used in AIDS vaccine development is relatively small when compared with the numbers that were used in the 1950s and 1960s in the development of poliovirus vaccines.

Animal models for AIDS are a crucially important resource with which to understand the biology of immunodeficiency viruses in vivo, and thus to gain essential insights into the mechanisms of pathogenesis and its prevention by vaccination and chemotherapy. In the twelve years since HIV was isolated, an impressive volume of knowledge has accumulated on the genetics and molecular biology of lentiviruses, but we remain largely ignorant of the biology of pathogenesis and strategies for prevention of this disease. In vitro experiments in cell culture may provide insights and clues, but there is no substitute for experiments in vivo, particularly with a virus that is so intimately involved with the complex cellular interactions of the intact immune system. Much has been learned about pathogenesis from the intensive study of HIV-infected patients. However, crucial early events in infection can only be investigated in animal models where timing, dose, route and nature of the challenge virus can be precisely controlled, and where the distribution of virus in a wide range of tissues can be assessed. Many antiviral therapies have been evaluated in clinical trials in infected volunteers. Nevertheless, animal models make a unique contribution to the evaluation of drugs administered immediately after exposure. Non-human primates also contribute to the evaluation of therapies involving immune modulators where the outcome is too uncertain to risk immediate use in humans. Vaccine strategies can only be assessed in vivo. Phase I and II human clinical trials have successfully tested the safety and antigenicity of candidate vaccines. However, phase III efficacy trials will be costly. Thus, efficacy data from animal models are crucial for identifying the in vitro immune correlates of protection and deciding which vaccines should proceed to phase III trials. Many candidate vaccines look promising until they are exposed to the harsh reality of a live virus challenge in vivo.

The use of chimpanzees remains controversial. They are a rare and protected species. Therefore, infected animals must be kept in containment for the duration of their natural life, and now require a pension fund in excess of 
US $\$ 100,000$ per individual. Although these animals are readily infected by certain strains of $\mathrm{HIV}-1$, replication is more limited than in humans and, so far, no animals have developed significant immunodeficiency disease. Hence, this model is inappropriate for the study of pathogenesis or the prevention of disease by vaccines or antiviral drugs. However, if the objective is to test the efficacy of a candidate HIV-1 vaccine against infection, then the chimpanzee is an appropriate animal model, and has been successfully used for this purpose. Vaccination with various HIV-1 envelope preparations and peptides has protected a number of chimpanzees against challenge with the homologous LAI strain ${ }^{4,5}$ or heterologous SF2 strain of HIV-1. Passive transfer of antibody to the $\mathrm{V} 3$ region of the envelope has also protected ${ }^{6}$. Caveats that must be observed in interpreting these results are (1) none of the individual experiments so far reported have reached statistical significance, (2) the challenge viruses are laboratory-adapted strains and (3) because the replication of HIV in chimpanzees is more restricted than in humans they may be more easily protected, and thus overestimate the efficacy of a vaccine designed for use in humans.

Infection of reconstituted SCID mice with HIV carries the same limitations as chimpanzees. Virus replication is limited and no immunodeficiency disease develops. In addition, the SCID mouse is a highly artificial animal. Despite these reservations the system has provided insights into the immunity of human volunteers vaccinated with HIV-1 vaccine whose serum or cells have subsequently been transferred to SCID mice, protecting a proportion against HIV-1 infection. It remains to be seen how far this elaborate organ culture system will predict the true efficacy of AIDS vaccines in humans.

Feline immunodeficiency virus infection of cats is a naturally occurring disease with its own economic importance. Hence, studies of pathogenesis and development of vaccines against this infection have their own intrinsic and commercial value, in addition to their value as a model for AIDS. Sufficient numbers of cats may be used to provide statistically significant results. However, the virus is significantly different from HIV-1 genetically, and it enters cells via a different receptor. The immune system of the cat is also substantially different from that of primates. Despite these limitations, insights obtained about FIV infection of cats and its prevention by vaccination are likely to be relevant to HIV infection in humans, and thus have added value to the need for a feline vaccine. Furthermore, if the same principles of pathogenesis and protection emerge from a variety of diverse animal models, confidence in their validity and relevance to human AIDS will be increased.

\section{The reality is that all animal}

$$
\text { models are potentially }
$$

capable of yielding relevant information, provided that experiments are appropriately designed and well controlled.

Macaque monkeys remain the most widely used animal model for AIDS. They can be infected by a variety of strains of SIV or HIV-2 which range from the highly virulent $\mathrm{PBj} 14$ strain of SIVsm to the attenuated infectious molecular clones of SIVmac carrying deletions in nef or other non-essential genes. Hence, precise studies of pathogenesis may be undertaken in which early events can be analysed in detail using a variety of viruses in which individual genes may be modified to define their role in virulence. Furthermore, vaccine strategies can be assessed for their ability to prevent virus infection, to reduce virus load, or to prevent or delay the development of disease. In all of these studies, adequate numbers of animals may be used to provide statistically significant data. SIV, like HIV, uses the CD4 receptor and is genetically closer — though not identical - to HIV-1 than any other vertebrate lentivirus. A further advantage of this model is that many of the human immunological markers and immune modulators are equally effective in the simian immune system.

The majority of macaques protected against infection were immunized with inactivated SIV ${ }^{7}$, but it is now clear that the protection was induced predominately by cellular antigens - not viral antigens - in the vaccine ${ }^{8}$. Vaccination of macaques with SIV envelope protein reduces virus load early after infection ${ }^{9,10}$ and may result in clearance of the infection and subsequent delay or prevention of disease. Complete protection against infection is only observed when the challenge virus has restricted replication in vivo as with SIVmne ${ }^{11}$.

By far the most potent protection so far observed in macaques is induced by attenuated virus ${ }^{12}$. Animals are protected against high doses of challenge virus given intravenously or intrarectally, and against challenge with intact virusinfected cells. The macaque is an ideal model in which to study attenuation of these viruses and the mechanisms by which they protect. A major limitation of the macaque model is that SIV and HIV-2 are not HIV-1, making it is a surrogate model. However, even this problem may be partially overcome by the use of chimeric viruses in which individual genes of SIV are replaced by those of HIV $-1^{13}$.

There are precedents for unwelcome results from the SIV macaque model. These were initially thought to be misleading, but they have helped to advance knowledge and to rectify generally held misconceptions. Some examples serve to illustrate this truth. First, early studies of the neutralization of HIV-1 laid great emphasis on the predominant importance of the V3 loop of the envelope protein. Since antibodies to the $\mathrm{V} 3$ region of SIV did not have neutralizing activity, the SIV macaque model was deemed to be irrelevant in HIV vaccine development. Subsequent studies on clinical isolates of HIV-1 suggest that the predominance of V3 may be an artefact of in vitro passage, indicating that the mechanism of neutralization is closer to that demonstrated for SIV than to the highly laboratory adapted IIIB strain of HIV-1 used in earlier studies. Second, the discovery in the SIV macaque model that T-cell antigens were capable of mediating protection against infection was initially condemned as a demonstration of the unreliability and irrelevance of this model in vaccine development. Subsequent studies have confirmed the observations and advanced our understanding of the intimate relationship between immunodeficiency viruses and their host cells. This discovery has also provided new opportunities for alternative approaches to AIDS vaccine development. Third, a series of in vitro studies on the role of the nef gene yielded conflicting results. The definitive in vivo studies by Desrosiers' group in the SIV macaque model established that nef is important in pathogenesis - a result subsequently confirmed by others ${ }^{12,14,15}$. 
All animal models have limitations but each will reveal essential insights that can only be obtained in vivo. The currently perplexing observations in this issue of Nature Medicine must have a logical explanation. Ultimately, we shall only know which is the best model when we understand the pathogenesis of AIDS, and when we have vaccines and therapies that prevent it. In the meantime, our own bias, on the evidence so far, is that infection of macaques with SIV or HIV-2 will prove to be the most rewarding and reliable model for pathogenesis and prevention of AIDS.

1. Abimiku A.G. et al. HIV-1 recombinant pox virus vaccine induces cross-protection against HIV-2 challenge in rhesus macaques. Nature Med. 1, 321-329 (1995).

2. Spouge J.L. Statistical analysis of sparse infection data and its implication for retroviral treatment trials in primates. Proc. natl. Acad. Sci. U.S.A. 89, 7581-7585 (1992)

3. The European Concerted Action on 'Macaque Models for AIDS Research'. Protection of macaques against simian immunodeficiency virus infection with inactivated vaccines: Comparison of adjuvants, doses and challenge viruses. Vaccine 13, 295-300 (1995).

4. Berman, P. et al. Protection of chimpanzees from infection with HIV-1 after vaccination with recombinant glycoprotein gp120 but not gp160. Nature 345, 622-625 (1990).

5. Fultz, P.N. et al. Vaccine protection of chimpanzees against challenge with HIV-1 infected peripheral blood mononuclear cells. Science 256, 1687-1690 (1992).

6. Emini, E. et al. Prevention of HIV-1 infection in chimpanzees by gp120 V3 domain specific monoclonal antibody. Nature $355,728-730$ (1992).

7. Warren, J.T. et al. First updated and revised survey of worldwide HIV and SIV vaccine challenge studies in non-human primates: Progress in first and second order studies. I. med. Primatol. 22, 203-235 (1993).

8. Stott, E.J. et al. Anti cell antibody in macaques. Nature 353, 393 (1991).

9. Ahmed S. et al. Reduced virus load in rhesus macaques immunized with recombinant gp160 and challenged with simian immunodeficiency virus. AIDS Res. hum. Retro. 10, 195-204 (1994).

10. Israel, Z.R. et al. Incomplete protection, but suppression of virus burden, elicited by subunit simian immunodeficiency virus vaccines. I. Virol. 68, 1843-1853 (1994).

11. Hu, S.L. et al. Protection of macaques against SIV infection by subunit vaccines of SIV envelope glycoprotein gp 160 . Science 255, 456-459 (1992).

12. Daniels, M.D. et al. Protective effects of a live attenuated SIV vaccine with a deletion in the nef gene. Science 258, 1938-1941 (1992).

13 .Shibata, R. et al. Generation of a chimeric human and simian immunodeficiency virus infectious to monkey peripheral blood mononuclear cells. $J$. Virol. 65, 3514-3520 (1991).

14. Kestler, H.W. et al. Importance of the nef gene for maintenance of high virus loads and development of AIDS. Cell 65, 651-662 (1991).

15. Rud, E. et al. Molecular and biological characterization of simian immunodeficiency virus macaque strain $32 \mathrm{H}$ proviral clones containing nef size variants. J. gen. Virol. 75, 529-543 (1994).

National Institute for Biological Standards and Control, Blauche Lane, South Mimms, Potters Bar, Hertfordshire EN6 3QG, UK

\section{Anatomical research lives!}

\section{A previously undescribed tissue connection may provide an explanation for some forms of tension headache. It also proves that common beliefs about anatomical research reaching its limits are unfounded.}

The announcement of a new anatomical finding is astonishing during these times of VAN P. THOMPSON

rative investigation by $\mathrm{Hack}$ and Robinson and their anatomical mentor Richard $\mathrm{T}$. re-evaluation and closing of anatomy departments in medical and dental schools. This is particularly true if such a finding helps explain the relationship between headache and muscle tension, a highly controversial area. Yet this has happened. At a recent meeting of the Joint Section on Disorders of the Spine and Peripheral Nerves of the American Association of Neurological Surgeons, Gary D. Hack of the University of Maryland at Baltimore Dental School and Walker L. Robinson of the University of Maryland School of Medicine (Baltimore) presented their findings on an observed connective tissue bridge between the spinal dura and the rectus capitis posterior minor (RCPM) Muscle (see figures). This observation, submitted for publication, represents over three years of collabotwo general dentists and a neurosurgeon.

Their finding was serendipitous. It occurred during inspection of the muscles of mastication in sagittally hemisected head and neck cadaveric specimens, which is not a standard dissection approach. The research team observed the RCPM muscledural connection and noted that simulated contraction of the muscle tensed the dura in this area. Subsequently they have found this connection in 25 consecutive dissections, including two black males, one black female, fourteen white males and eight white females. A suitable animal model is being actively sought. This connective tissue bridge has been verified by James $\mathrm{L}$. Hiatt, an anatomist at the University of Maryland at Baltimore,
Koritzer, a unique collaboration between

\section{IMAGE UNAVAILABLE FOR COPYRIGHT REASONS}

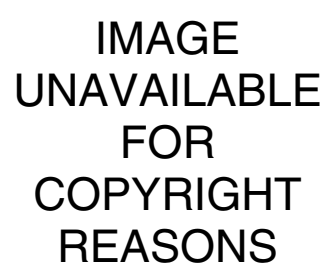

Illustrations of the region in the head where a previously undiscovered muscle-dural connection is found. (above) A drawing of the deep occipital region (posterior view). The rectus capitis posterior minor (RCPM) muscle is seen extending from the first cervical vertebrae $(\mathrm{C} 1)$ to the occipital bone. Superficial musculature has been removed.

(left) A drawing of a hemisected head and neck specimen. 1, occipital bone (posterior border of foramen magnum); 2, atlas (posterior arch); 3, RCPM muscle; 4 , dura mater; 5 , cerebrospinal fluid in cisterna magna; 6 , spinal cord. Arrow, connective tissue bridge linking the RCPM muscle to the dura mater. 